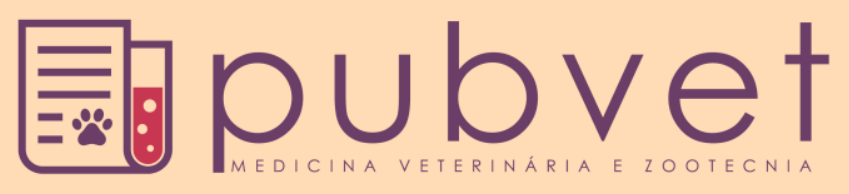

https://doi.org/10.22256/pubvet.v12n5a88.1-4

\title{
Acetato de deslorelina como agente indutor de ovulação em éguas
}

\author{
Eduardo Herrera Dias ${ }^{0}$, Taniara Suelen Mezalira ${ }^{\bullet}$, Adalgiza Pinto Neto ${ }^{\bullet}$, Luan \\ Sitó Da Silva ${ }^{\bullet}$, Stella Maris Teobaldo Tironi ${ }^{\circ}$, Anna Raquel Grimas Almeida ${ }^{\circ}$, \\ Antonio Campanha Martinez ${ }^{\circ}$ 2* $^{*}$
}

${ }^{I}$ Curso de Medicina Veterinária da Universidade Estadual de Maringá, Departamento de Medicina Veterinária . Umuarama-PR Brasil

${ }^{2}$ Programa de Pós-Graduação em Produção Sustentável e Saúde Animal. Universidade Estadual de Maringá, Umuarama-PR Brasil

${ }^{3}$ Curso de Medicina Veterinária da Universidade Federal da Fronteira Sul. Realeza-PR Brasil

*Autor para correspondência: acmartinez@uem.br

RESUMO. As éguas são animais reprodutivos estacionais, sendo influenciadas pelas mudanças na relação diária de luminosidade durante o ano, apresentando uma maior dificuldade no controle do ciclo estral, quando comparado aos bovinos. A utilização de indutores de ovulação pode melhorar a eficiência reprodutiva, sincronizando o momento das ovulações e reduzindo o período do estro. Os objetivos deste estudo foi avaliar o uso de indução do estro estimulado por implante de progesterona e o tempo de ovulação em fêmeas equinas utilizando o acetato de deslorelina como indutor de ovulação. Foram utilizadas cinco éguas em idade reprodutiva, que tiveram seu estro induzido por duas vezes cada uma. Em uma das vezes nas mesmas foi administrada com $750 \mathrm{mg}$ de acetato de deslorelina. Para indução do estro foi aplicado uma dose de $5 \mathrm{mg}$ via IM de prostaglandina F2 no D0 e inserido um dispositivo intra-vaginal que foi removido no D8, sendo concomitante a remoção aplicado uma dose de prostaglandina. Após a remoção dos dispositivos vaginais avaliou-se o crescimento folicular em intervalos de 12/12h até a ovulação. Todos os animais demonstraram comportamento de estro. Éguas tratadas com acetato de deslorelina tiveram o tempo de ovulação em média de $43,2 \mathrm{~h} \pm 6,57$ enquanto o grupo não tratado foi de $84 \mathrm{~h} \pm 14,70$, apresentando diferença significativa. Podendo-se concluir que o uso dispositivo intra-vaginal para bovinos induz a manifestação do estro em éguas; a administração de acetato de deslorelina antecipa o tempo de ovulação contribuindo para um melhor manejo reprodutivo em éguas.

Palavras chave: cio, equina, ovário, reprodução

\section{Deslorelin acetate to induce ovulation in mares}

ABSTRACT. The mares are seasonal reproductive animals, being influenced by the changes in the daily relation of luminosity during the year, presenting a greater difficulty in the control of the estrous cycle when compared to the cattle. The use of ovulation inducers can improve reproductive efficiency by synchronizing the time of ovulation and reducing the period of estrus. The objectives of this paper were to evaluate the use of progesterone-device for cow estrous induction and ovulation time in mares using deslorelin acetate as an ovulation inducer. Five mares of reproductive age were used, which had their estrus induced twice each. In one of them it was administered with $750 \mathrm{mg}$ of deslorelin acetate. For estrous induction, a 5mg dose was administered via IM of prostaglandin F2alpha at the D0 and an intravaginal device was inserted, which was removed at D8, and a dose of prostaglandin was administered concomitantly. After removal of the vaginal devices follicular growth was evaluated at $12 / 12 \mathrm{~h}$ intervals until ovulation. All animals showed estrus behavior. Mares treated with deslorelin acetate had a mean ovulation time of $43.2 \pm 6.57$ while the untreated group was $84.0 \pm 14.70$, with a significant difference. It 
can be concluded that the intra-vaginal device use for cattle induces the manifestation of estrus in mares; The administration of deslorelin acetate anticipates the time of ovulation contributing to a better reproductive management in mares.

Keywords: equine, estrous, ovary, reproduction

\section{Acetato de deslorelina como agente inductor de ovulación en yeguas}

RESUMEN. Las yeguas son animales reproductivos estacionales, siendo influenciadas por los cambios en la relación diaria de luminosidad durante el año, presentando una mayor dificultad en el control del ciclo estral, cuando comparado a los bovinos. La utilización de inductores de ovulación puede mejorar la eficiencia reproductiva, sincronizando el momento de las ovulaciones y reduciendo el período del estro. Los objetivos de este estudio fueron evaluar el uso de inducción del estro estimulado por implante de progesterona y el tiempo de ovulación en hembras equinas utilizando el acetato de deslorelina como inductor de ovulación. Se utilizaron cinco yeguas en edad reproductiva, que tuvieron su estro inducido dos veces cada una. En una de las veces en las mismas fue administrada 750mg de acetato de deslorelina. Para la inducción del estro se aplicó una dosis de $5 \mathrm{mg}$ vía IM de prostaglandina F2 en el D0 e insertado un dispositivo intra-vaginal que fue removido en el D8, siendo concomitante la remoción aplicada una dosis de prostaglandina. Después de la remoción de los dispositivos vaginales se evaluó el crecimiento folicular a intervalos de 12 / 12h hasta la ovulación. Todos los animales demostraron comportamiento de estro. Las yeguas tratadas con acetato de deslorelina tuvieron el tiempo de ovulación en promedio de $43,2 \mathrm{~h} \pm 6,57$ mientras que el grupo no tratado fue de $84 \mathrm{~h} \pm 14,70$, presentando diferencia significativa. Se puede concluir que el uso dispositivo intra-vaginal para bovinos induce la manifestación del estro en yeguas; la administración de acetato de deslorelina anticipa el tiempo de ovulación contribuyendo a un mejor manejo reproductivo en yeguas.

Palabras clave: Celo, equina, ovario, reproducción

\section{Introdução}

Durante o ciclo estral as éguas apresentam grande variação de animal para animal no tempo entre o início do cio até o momento da ovulação variando de 2 até 14 dias (Mccue 2003) e no tamanho dos folículos ovulatórios, podendo ser de $35 \mathrm{~mm}$ até $\geq 50 \mathrm{~mm}$ (Samper 1997). Apesar dos animais apresentarem esta variação fisiológica, para se obter maiores taxas de concepção o sêmen deve ser depositado no trato reprodutivo da égua em um momento mais próximo possível da ovulação (Kölling \& Allen 2006; Greco et al. 2016).

A utilização de indutores de ovulação pode melhorar a eficiência reprodutiva, reduzindo o período do estro e sincronizando o momento das inseminações, consequentemente diminuindo gastos com transporte, otimizando as doses de sêmen e o uso do garanhão (Melo et al. 2012). A utilização rotineira de indutores de ovulação como o hCG provoca uma diminuição em sua eficácia devido a formação de anticorpos contra estes agentes exógenos, no entanto os análogos de GnRH estimulam a ovulação pela liberação de LH sem a formação de anticorpos (Greco 2010). O desenvolvimento de GnRH sintético fez com que se aumentasse a meia vida deste hormônio pelas modificações na estrutura do GnRH natural, permitindo um aumento nos níveis de LH após sua administração e reduzindo a duração do estro, assim, vários estudos têm sido feitos para avaliarem seu uso no controle do ciclo estral de éguas (Bergfelt 2000). Um dos análogos de GnRH é a deslorelina, que no início era utilizada na forma de implantes subcutâneos na presença de folículos com diâmetro $\geq 30 \mathrm{~mm}$ para induzir a ovulação, no entanto o uso deste dispositivo gerou um aumento indesejável do intervalo entre os ciclos estrais. Já a deslorelina injetável não produz este efeito indesejável, levando à ovulação, na maioria das vezes, em até 48 horas (Boakari 2014).

Os objetivos deste trabalho foi avaliar o uso de indução do estro estimulado por implante de progesterona e tempo de ovulação em fêmeas equinas utilizando o acetato de deslorelina como indutor de ovulação.

\section{Material e Métodos}

O experimento foi realizado no Laboratório de Criação e Reprodução Animal da Universidade Estadual de Maringá, Campus Umuarama, 
utilizando-se cinco éguas em idade reprodutiva. Exames ultrassonográficos diários foram realizados do início do tratamento até o momento da ovulação. Foi aplicado uma dose de $5 \mathrm{mg}$ via IM de prostaglandina F2 $\alpha\left(\right.$ Lutalyse $\left.^{\circledR}\right)$ no D0 e inserido um dispositivo intra vaginal $\left(\mathrm{CIDR}^{\circledR}\right)$ que foi removido no D8, sendo concomitante a remoção aplicado uma dose de prostaglandina. Após isso as éguas foram divididas em dois tratamentos, no qual $\mathrm{T} 1$ avaliou o crescimento folicular em intervalos de 12/12h até a ovulação e $\mathrm{T} 2$ avaliou o crescimento folicular em intervalos de $12 / 12 \mathrm{~h}$, assim que o folículo atingiu diâmetro médio de $>35 \mathrm{~mm}$, foi administrado uma dose de $750 \mu \mathrm{g}$ de acetato de deslorelina (Sincrorrelin ${ }^{\circledR}$ ) por via IM, e feito o acompanhamento ultrassonográfico pós-aplicação, em intervalo de 12 horas, até confirmada a ovulação. Uma semana após a ovulação os animais foram novamente submetidos ao tratamento previamente descrito com 5mg via IM de prostaglandina F2 $\alpha$ (Lutalyse $\AA$ ) no D0 e reinserido um dispositivo intra-vaginal $(C I D R \AA)$ que foi removido no D8, sendo concomitante a remoção aplicado uma dose de prostaglandina. E desta vez foram novamente acompanhados com auxílio de exames de ultrassonografia ovariana em uma distribuição em cross over, onde os que participaram do $\mathrm{T} 1$ foram tratados como o T2 e vice-versa.

\section{Resultados e Discussão}

O protocolo de indução do estro utilizado teve uma eficácia de $100 \%$. Na primeira etapa, quanto na segunda todas as éguas exibiram comportamento de estro, sendo, portanto, eficaz a utilização de implantes de progestágenos, indicados para bovinos, para a indução do estro de éguas. Obteve-se uma taxa de $100 \%$ de ovulação em até 48 horas no grupo de éguas tratadas com o acetato de deslorelina. No grupo não tratado levou até 108 horas para ocorrer a ovulação após a detecção de um folículo $\geq 35 \mathrm{~mm}$ (Figura 1).

Em relação ao diâmetro dos folículos, no grupo tratado a ovulação ocorreu com 38,46 \pm 17,76; enquanto que no grupo não tratado a ovulação o ocorreu com folículos de 39,26 $\pm 8,09$, apresentado um valor de $\mathrm{p}=0,737$ (Figura 1). Houve diferença $(\mathrm{P}<0,01)$ em comparação ao tempo de ovulação entre o grupo tratado e o não tratado, sendo de $43,2 \mathrm{~h} \pm 6,57$ e de $84 \mathrm{~h} \pm 14,70$ respectivamente.

O entendimento do mecanismo de ação dos indutores de ovulação é de suma importância para sua indicação. $\mathrm{O}$ uso de progestágenos na sincronização do estro auxilia uma melhor uniformidade na sua manutenção. Segundo Martinez et al. (2017) o uso de implante vaginal bovino causa incomodo mínimo às éguas, sendo indicado o seu uso, com a mesma conclusão Polasek et al. (2017) recomendam o uso de implantes vaginais bovinos para a indução do estro, porém estes autores trabalharam com animais acíclicos no período de transição.

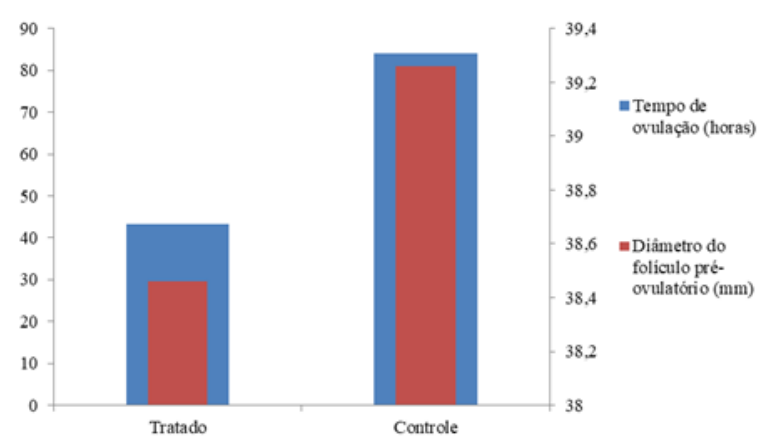

Figura 1. Tempo de ovulação, em horas, e o diâmetro folicular de éguas submetidas, ou não, ao acetato de deslorelina com agente indutor de ovulação.

A deslorelina é um agonista sintético do GnRH, estimulando a liberação prolongada de LH e FSH da pituitária anterior, dessa forma induzindo a ovulação (McCue et al. 2007). Neste trabalho $100 \%$ dos animais tratados com acetato de deslorelina ovularam em até 48 horas, resultados semelhantes foram obtidos por $\mathrm{McCue}$ et al. (2007) e Ferris et al. (2012), onde 91,1\% e $89,9 \%$ dos animais tratados com deslorelina ovularam até 48 horas respectivamente. Este resultado pode ser devido ao número de animais utilizados ou pelo tamanho dos folículos no momento da aplicação.

As éguas do grupo tratado e não tratado foi em média de $43,2 \mathrm{~h} \quad \pm 6,57$ e $84 \mathrm{~h} \quad \pm 14,70$ respectivamente, apresentando diferença $(\mathrm{P}<$ $0,01)$. Tais resultados corroboram com os obtidos por Melo et al. (2012), no qual as ovulações concentraram-se entre 36 e 48 horas após a utilização do acetato de deslorelina, e mostrando que o uso do acetato de deslorelina antecipa o momento da ovulação. Essa antecipação pode ser uma excelente alternativa para práticas de campo, uma vez que desta maneira determina um menor tempo de serviços com estes animais.

O grupo tratado teve a ovulação com folículo com diâmetro médio de 38,46 $\pm 17,76 \mathrm{~mm}$, já o grupo não tratado a ovulação ocorreu com $39,26 \pm$ $8,09 \mathrm{~mm}$. Apesar do grupo tratado ovular com 
diâmetro numericamente menor, não houve diferença $(P>0,05)$ entre os grupos. Resultados semelhantes foram relatados no trabalho realizado por Ferris et al. (2012), onde a ovulação ocorreu com $39,9 \pm 4,5 \mathrm{~mm}$ nos animais tratados com deslorelina.

\section{Conclusão}

Com base nos resultados obtidos neste estudo foi possível concluir que o uso de dispositivo intra-vaginal para bovinos induz a manifestação do estro em éguas. $\mathrm{E}$ a utilização de acetato de deslorelina antecipa o tempo de ovulação contribuindo para um melhor manejo reprodutivo em éguas.

\section{Referências Bibliográficas}

Bergfelt D. R. 2000. Estrous synchronization. mare. Saunders, Philadelphia, USA.

Boakari Y. L. 2014. Efeito da hCG ou deslorelina sobre a hemodinâmica folicular e perfil endogeno de LH em éguas cíclicas. In: Faculdade de Medicina Veterinária $e$ Zootecnia. Universidade Estadual Paulista, Botucatu, São Paulo.

Ferris R. A., Hatzel J. N., Lindholm A. R. G., Scofield D. B. \& McCue P. M. 2012. Efficacy of deslorelin acetate (SucroMate) on induction of ovulation in American Quarter Horse mares. Journal of Equine Veterinary Science 32, 2858.

Greco G. M. 2010. Avaliação de novos protocolos visando induzir e sincronizar a ovulação em éguas. In: Faculdade de Medicina Veterinária e Zootecnia. Universidade Estadual Paulista, Botucatu, São Paulo.

Greco G.M., Fioratti E.G., Segabinazzi L.G., Dell'Aqua J.A., Crespilho A.M., CastroChaves M.M.B. \& Alvarenga M.A. 2016. Novel long-acting progesterone protocols used to successfully synchronize donor and recipient mares with satisfactory pregnancy and pregnancy loss rates. Journal of Equine Veterinary Science 39, 58-61.

Kölling M. \& Allen W. R. 2006. Ovulation induction for embryo transfer: hCG versus
GnRH analogue. In: Proceedings of a Workshop, Havemeyer Foundation Monograph Series, pp. 54-5.

Martinez A. C., Colli M. H. A., Carvalho R. S., Moleirinho J.O., Ruivo M. A., Neto A. P. \& Lopes W. D. 2017. Comportamento de éguas após a inserção de dispositivo intravaginal impregnado com progesterona. Arquivos de Ciências Veterinárias e Zoologia da UNIPAR 19, 143-6.

Mccue P. M. 2003 Induction of ovulation. In: Current therapy in equine medicine (ed. by Rpbinson NE), pp. 240-2. Saunders, Philadelphia, USA.

McCue P. M., Magee C. \& Gee E. K. 2007. Comparison of compounded deslorelin and hCG for induction of ovulation in mares. Journal of Equine Veterinary Science 27, 5861.

Melo C. M., Papa F. O., Puoli Filho J. N. P., Araujo G.H.M., Dell'Aqua Junior J. A. \& Alvarenga M.A. 2012. Eficiência do acetato de deslorelina e do extrato de pituitária equina na indução da ovulação em éguas. Veterinária $e$ Zootecnia 19, 392-8.

Polasek T. C. M., Kozicki L. E., Pedrosa V. B., Weiss R. R., Bertol M. A. F., Camargo C. E. \& Talini R. 2017. Impact of a progesteronereleasing intravaginal device and inflammatory reaction on ovarian activity in embryorecipient anestrus mares. Theriogenology 90, 175-84.

Samper J.C. 1997. Ultrasonographic appearance and the pattern of uterine edema to time ovulation in mares. In: Proceedings of the 43rd Annual Convention of the American Association of Equine Practitioners, pp. 18991.

\section{Article History:}

Received 2 February 2018

Accepted 2 April 2018

Available online 7 May 2018

License information: This is an open-access article distributed under the terms of the Creative Commons Attribution License 4.0, which permits unrestricted use, distribution, and reproduction in any medium, provided the original work is properly cited. 